\title{
Recognition of a Fetal Subdiaphragmatic Venous Vestibulum Essential for Fetal Venous Doppler Assessment
}

\author{
TJEERD W. A. HUISMAN, ADRIANA C. GITTENBERGER-DE GROOT, AND \\ JURIY W. WLADIMIROFF \\ Department of Obstetrics and Gynecology Academic Hospital Rotterdam-Dijkzigt, Erasmus University, \\ Rotterdam [T.W.A.H., J.W.W.] and Department of Anatomy and Embryology University of Leiden, \\ Leiden, the Netherlands [A.C.G-de G.]
}

\begin{abstract}
UItrasonic visualization of the human fetal subdiaphragmatic area demonstrated anatomical relationships, different from descriptions in the literature. Four human fetal postmortem specimens at 18, 26, 28, and 34 wk of gestation were examined to ascertain morphologic details of intra- and perihepatic vasculature. Drawings of these dissected preparations were compared with ultrasonic images from the same region. With both methods the presence of a venous vestibulum immediately proximate to the diaphragm could be demonstrated. The abdominal inferior vena cava ends in a funnel-like structure, which also contains the orifices of the hepatic veins, the ductus venosus, and a phrenic vein. A considerable variability in Doppler flow recordings could result from blood propelling out of these various vessels into the vestibulum. It is, therefore, suggested that information on blood-flow velocities in venous hepatic vessels should be obtained more distally in the separate vessels and not at the entrance into the right atrium. (Pediatr Res 32: 338-341, 1992)
\end{abstract}

Abbreviations

US, umbilical sinus

Ip, left portal vein

rp, posterior branch right portal vein

ra, anterior branch right portal vein

RA, right atrium

IVC, inferior vena cava

DV, ductus venosus

VHD, right hepatic vein

Recently, a sonographic method to obtain Doppler velocity wave forms from the human fetal IVC by ultrasonic guidance was described (1). The proposed site to direct the Doppler beam is situated just proximate to the RA. However, visualization of this area by two-dimensional real-time ultrasound demonstrated anatomical relationships that did not correlate with morphologic descriptions in the literature (2-7). The IVC is considered to course straight through the diaphragm into the RA, although other veins, such as the DV and the hepatic veins, discharge separately into this straight vessel at different locations along its subdiaphragmatic course through and above the fetal liver (47).

Received January 13, 1992; accepted April 24, 1992.

Correspondence: J. W. Wladimiroff, M.D., Ph.D., Professor of Obstetrics and Gynecology, Department of Obstetrics and Gynecology, Academic Hospital Rotterdam-Dijkzigt, Dr. Molewaterplein 40, 3015 GD Rotterdam, The Netherlands.
During Doppler studies of the IVC immediately proximate to the RA, other flow velocity wave forms often were encountered, in particular from the left hepatic vein and DV. Also an unexplained large $\mathrm{SD}$ was found while analyzing IVC wave form parameters from this site (8). Two studies suggest a clinical importance of these parameters in cases of arrhythmias and growth retardation $(1,9)$.

The aim of the present study was to ascertain the exact anatomical relationship between IVC, DV, and the hepatic veins in the human fetus in the diaphragmatic and subdiaphragmatic area.

\section{MATERIALS AND METHODS}

Postmortem specimens of four human fetuses at 18, 26, 28 and $34 \mathrm{wk}$ of gestation, taken at random, were examined.

The abdominal cavity had been opened in all fetuses during autopsy, and in three fetuses heart and lungs had been removed immediately superior to the diaphragm to exclude cardiac anomalies. No macroscopically detectable congenital abnormalities were found.

The umbilical vein served as a guide for the exposure of the hepatic vasculature. After the removal of surrounding liver parenchyma, the blood vessels in the hepatic region around and above the intra-abdominal part of the umbilical vein were carefully dissected, so they could be traced to their origin. Drawings were made of two of four specimens (Figs. 1-3). Interest was particularly focused on the continuity of the hepatic vasculature. No details are given about the relationship between hepatic vessels and hepatic ligaments and lobulation, because these structures cannot be detected with real-time ultrasound.

Next, the anatomical situation at the level of the diaphragm was assessed. An incision was made through the diaphragm and the subdiaphragmatic region in all four specimens (Figs. 1 and $3)$.

These preparations demonstrate anatomical details in a threedimensional way; ultrasound images from the same area are only a two-dimensional visualization. Nevertheless, sonographic images with enough details were obtained to compare these with the anatomical drawings.

One image (Fig. 4) was made from a normal 28-wk-old fetus in a sagittal scanning plane, in which the US with the arising DV in the liver served as a landmark. The fetal spine was situated posteriorly. The second ultrasound image is from a normal 36wk-old fetus in a transverse to oblique view (Fig. 5). In this case, the fetal portal vasculature in the liver was visualized in relation to the IVC, DV, and the RA, while again the US served as the landmark anteriorly in the fetal abdomen. 


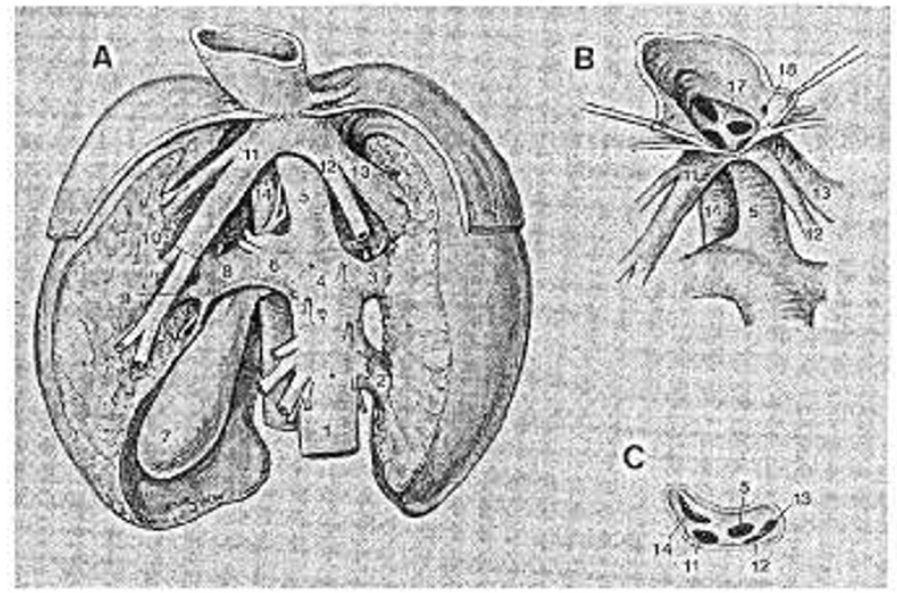

Fig. 1. Drawing of a postmortem prepared normal human fetus at 34 wk of gestation; anterior view of the liver and diaphragm: $A$, complete preparation; $B$, detail of the subdiaphragmatic area with incision through the diaphragm; $C$, cranial view from $B$. Legend to Figures 1,2 , and $3: 1$, umbilical vein; 2 , inferolateral branch of the $\mathrm{lp} ; 3$, superolateral branch of the lp; 4, umbilical part of the $\mathrm{lp} ; 5, \mathrm{DV} ; 6$, transverse part of the $\mathrm{lp}$, 7 , gallbladder; 8 , right portal vein; 9 , anterior branch of the right portal vein; 10 , posterior branch of the right portal vein; 11 , VHD; 12 , hepatic vein; 13 , left hepatic vein; 14, IVC; 15, gastric vein; 16 , portal vein; 17 , venous vestibulum; 18, phrenic vein; 19, esophagus; 20 , afferent hepatic veins.

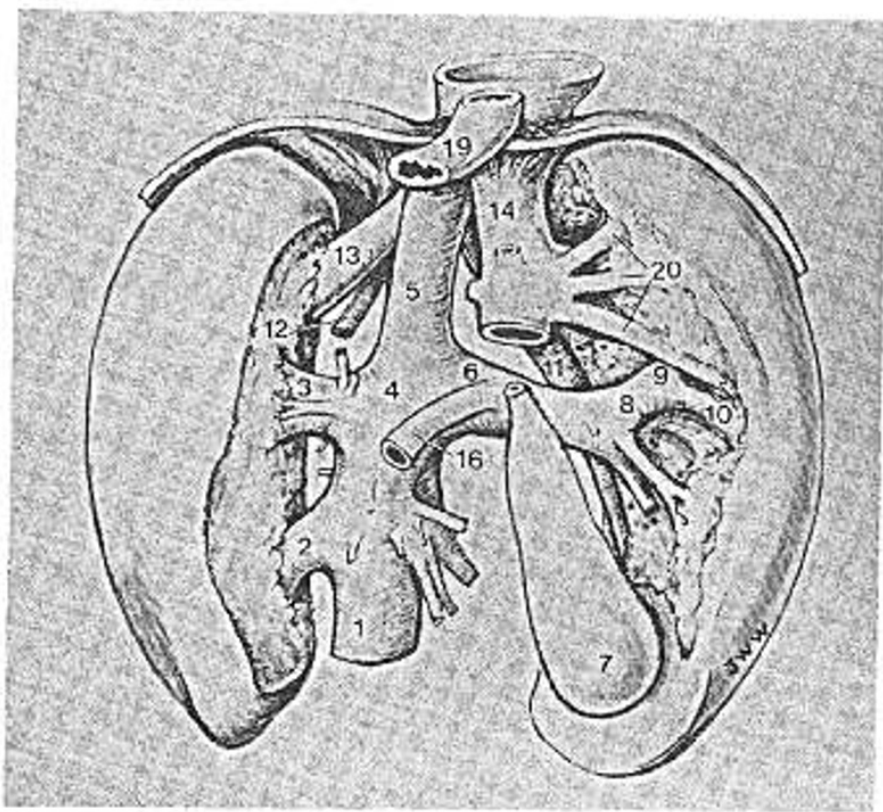

Fig. 2. Drawing of a postmortem prepared normal human fetus at 34 wk of gestation; posterior view of the liver and diaphragm.

\section{RESULTS}

Anatomical data. The left hepatic lobe is almost as large as the right lobe. The umbilical vein courses posteriorly and slightly cephalically and somewhat to the left of the midline between the right and left hepatic lobes (Fig. 1). No side branches were detected before its termination into the lp. Several small branches, leading to medial and lateral segments of the left hepatic lobe, are present in the umbilical portion of the $1 \mathrm{p}$. Two large veins running to the left arise from this portion, which have been identified as the superolateral and the inferolateral branches of the umbilical segment of the $\operatorname{lp}(2)$. The gallbladder is situated more laterally to the right. The course of the $\mathrm{lp}$ then takes an

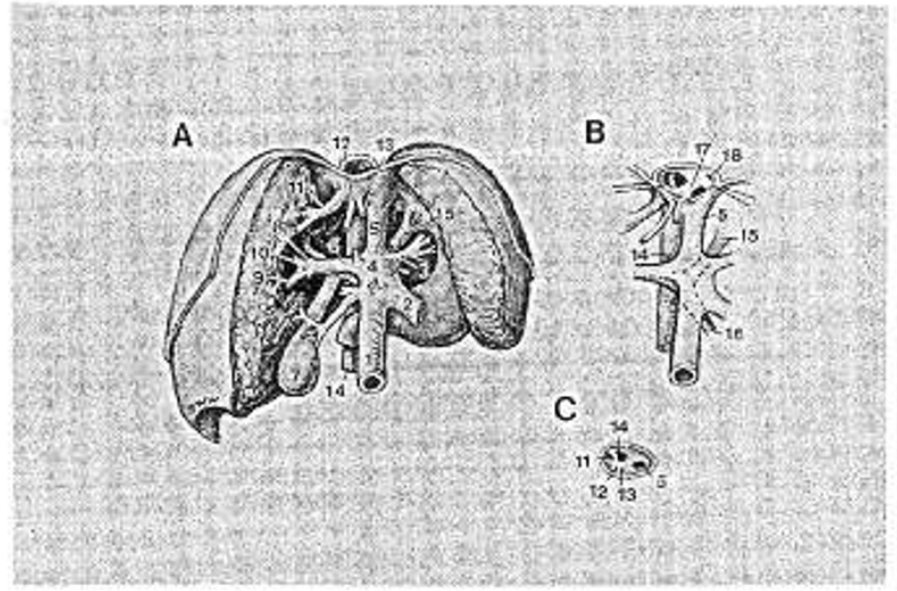

Fig. 3. Drawing of a postmortem prepared normal human fetus at 18 wk of gestation; anterior view of the liver and diaphragm. $A$, complete preparation; $B$, detail of the subdiaphragmatic area with incision through the diaphragm; $C$, cranial view from $B$.

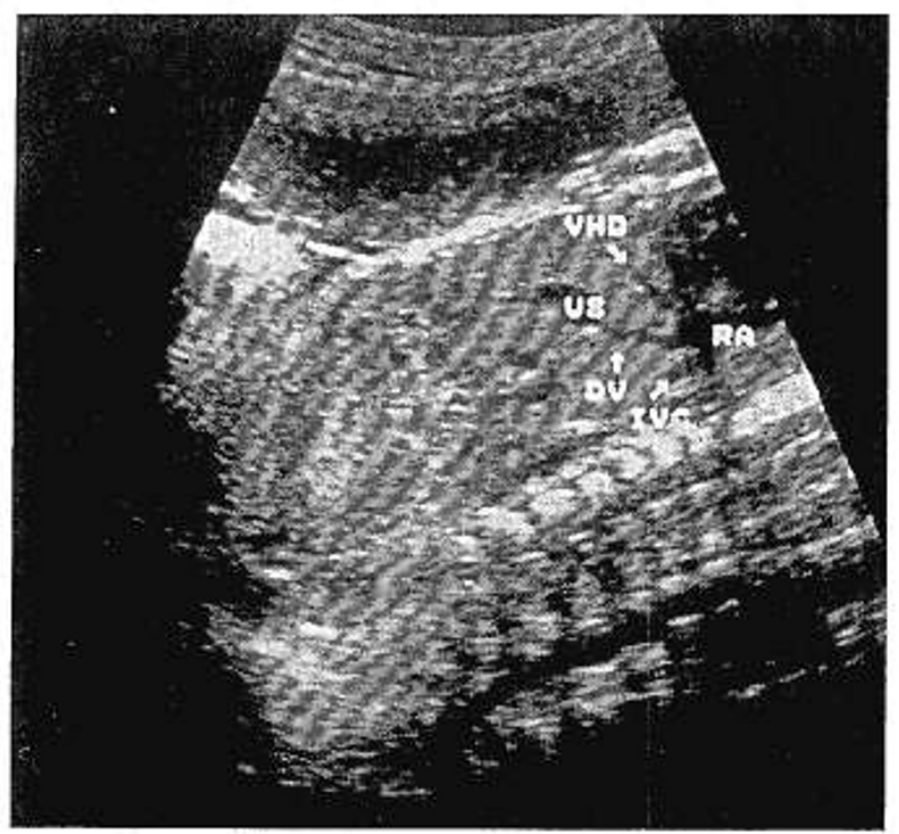

Fig. 4. Ultrasonic image from a normal human fetus at 28 wk gestation in a sagittal scanning plane. The posteriorly situated fetal spine and the US serve as landmarks.

abrupt right turn, running in front of the ductus cysticus, to form the transverse part of this vessel. At the turn a large vessel arises anteriorly out of this transverse part coursing superoposteriorly, slightly left oblique and more cranial: this is the DV. It has no side branches and continues in the direction of the diaphragm and RA. The transverse part of the lp is continuous with the right portal vein. The right portal vein divides into an anterior and posterior branch. In a posterior view, the common portal vein can be visualized behind the umbilical vein (Fig. 2). It connects with the transverse part and runs in the direction of the right portal vein. Also, the IVC receives posteriorly three small hepatic veins from the right liver lobe.

The anterior view shows that multiple veins arise from the right as well as from the left hepatic lobe. These veins confluence and flow into a left, middle, and right hepatic vein (Fig. 1). All three vessels course in the direction of the RA, which is situated just above the diaphragm. Left and sagittal (or middle) hepatic veins cross anterior to the DV just below the diaphragm. The 


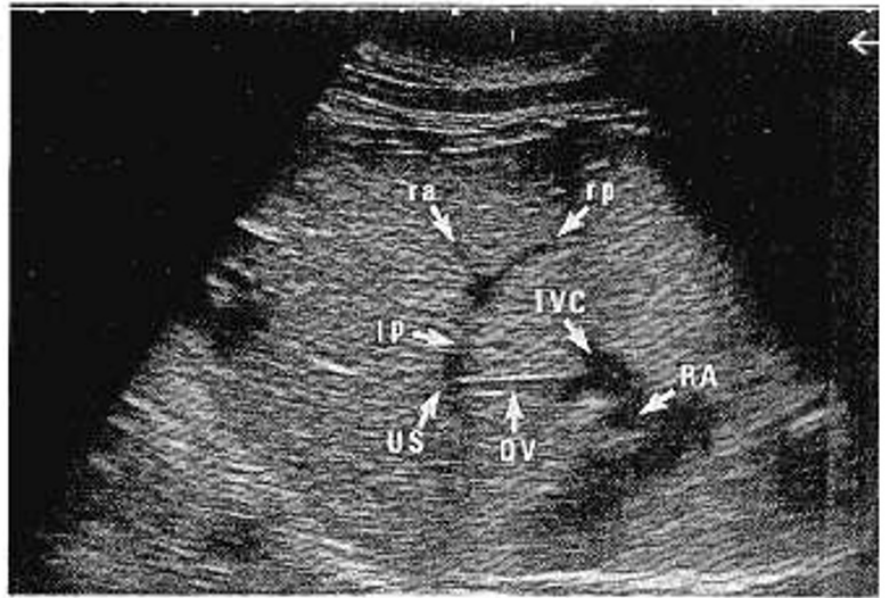

Fig. 5. Ultrasonic image from a normal human fetus at $36 \mathrm{wk}$ gestation in a transverse to oblique scanning plane. Landmarks are the anteriorly situated US and the lp.

exact individual vessel relationship is shown by the dissection, in which the diaphragm has been incised (Fig. 1). The various vessel orifices all enter a funnel-like cavity situated just below the diaphragm. This funnel (vestibulum) receives the abdominal part of the IVC, the DV, and the orifices of the hepatic veins. In the 18-wk specimen and in the 34-wk specimen there was a separate orifice for a phrenic vein (Figs. 1 and 3). The vestibulum continues through the diaphragm where it connects the RA as the thoracic part of the IVC.

The anatomical relationship between these venous vessel structures is not essentially different when comparing 18-wk and 34wk-old fetuses (Figs. 1 and 3). One observation, however, concerns the change in diameter of the hepatic veins relative to the IVC and DV with advancing gestational age. In the 18-wk-old fetus (Fig. 3), the diameter of the hepatic veins is only approximately one fifth of that measured at the IVC and DV, although this ratio has increased to approximately half in the 34-wk-old fetus (Fig. 1).

Sonographic data. The sagittal ultrasound scan from the 28wk fetus indicates that the DV, the VHD, and the IVC confluence at the level of the diaphragm before entering the RA (Fig. 4). The VHD seems to have approximately the same diameter as the DV, although the US and the IVC demonstrate slightly wider vessel sizes. Examination of the 36-wk fetus shows a similar situation (Fig. 5). The DV arises from the US, which is an important landmark for measurement of the fetal abdomen circumference (10), to join the IVC. At that site, a clear widening of the venous structure can be detected before its termination in the RA. This image also gives a well-defined sonographic visualization of the fetal portal vasculature. The right portal vein, which is continuous with the $\mathrm{lp}$, splits into an anterior and a posterior branch.

No essential differences can be detected when comparing the drawings of the prepared specimens with the ultrasound images. The limitation of ultrasonic diameter measurements does not allow exact comparison between anatomically obtained vessel sizes and sonographic data.

\section{DISCUSSION}

Study of the venous distribution in the subdiaphragmatic area in the dissected specimen showed a different morphology as described in the literature (2-7). In intra-uterine life the fetal IVC does not simply continue from the abdomen to the thorax but demonstrates a change in structure just below the diaphragm. The abdominal IVC ends in a funnel-like venous structure, which contains the orifices of the hepatic veins as well as the DV. This subdiaphragmatic vestibulum is interposed between the abdominal and thoracic part of the IVC. The term subdiaphragmatic vestibulum is used for clarity, although part of the venous structure is situated at the level of the diaphragm.

For the developmental background, it is important to know that an extensive remodeling of the venous vasculature takes place in the human embryonic period. This is nicely depicted in advancing developmental stages by Gilbert (6). In the 4th wk of gestation, the primitive venous system consists of three sets of symmetrically paired veins: the vitelline, the umbilical, and the pre- and postcardinal veins. The vitelline veins return blood from the yolk sac and the gut and form the connection with developing hepatic sinusoids and the heart as the hepatocardiac channels. These will form in a process, in which part of the venous system disappears while other parts anastomose the portal vein plus side branches. By the 6th wk the suprahepatic portion of the IVC consists of a persisting portion of the right hepatocardiac channel. This hepatocardiac channel comes closest to the vestibulum described here, but in the literature it is seen as the IVC (6).

In embryonic life the sinus venosus, situated in the thorax, receives blood from all three sets of paired veins, but during the 5 th wk of gestation it is incorporated into the dorsal part of the right atrium, while the veins are remodeled into the inferior and superior vena cava, DV, and the portal system by radical transformations $(5,6)$. In the adult, the remnants of the sinus venosus are present in the right atrium as the sinus venarum and the coronary sinus.

In textbooks it is presumed that the abdominal IVC, once constructed by anastomosis of parts from the right sacrocardinal, subcardinal, and vitelline veins, becomes the main vessel that directs blood to the right atrium (5-7). In our opinion, it remains an afferent vessel like the DV and the hepatic veins, confluencing into a subdiaphragmatic vestibulum, which forms the final connection with the RA as the thoracic IVC. Only when the DV and the umbilical vein blood supply are interrupted after delivery can the IVC become the straight main blood vessel, returning deoxygenated blood from the lower body to the right heart. Until now the anatomy of the venous vestibulum as such has not been appreciated or recognized. Rather, it is described, at that level, as a dilatation of the terminal part of the IVC or as a variation in entrance of the various veins into the IVC or into the hepatic veins $(7,12)$. In an elegant ultrasonic evaluation, it is demonstrated that various veins enter the IVC just below the diaphragm, but the exact anatomical morphology is not shown (3).

With development of gestation there is a marked increase in the dimensions of the hepatic venous return into this vestibulum. It is presumed that the hepatic erythropoiesis is mainly responsible for changes in fetal liver size (13). Whether our observation that hepatic afferent veins seem to increase relatively in vessel size during advancing gestation can be explained by this development in fetal hemopoiesis remains unclear.

In both the 18-wk and 34-wk-old fetus a separate orifice for a phrenic vein could be distinguished in the vestibulum. We could not differentiate, in consulting an anatomical textbook (7), whether it concerns the superior or inferior phrenic vein, although this information has little clinical relevance.

For the clinical evaluation, however, it is important that the presence of the observed vestibulum implies the data obtained prenatally by Doppler assessment at the inlet of the right atrium provide information on changes in general venous return rather than information on IVC blood flow alone. Moreover, a considerable variability in flow recording could result from the influence of blood flow from the various vessels propelling into the vestibulum. It was proposed in fetal sheep that blood streams with negligible mixing via the venae cavae and DV into the right atrium (11). One can imagine that small changes in scanning plane (such as may be the case during fetal, maternal, or examiner movements) may cause variation in blood flow measurements at the site of this vestibulum. This has been observed in a previous study (8).

In conclusion, it is suggested that information on blood flow velocities in the hepatic and subdiaphragmatic area should be 
obtained more distally in the separate vessels and not at the venous entrance into the RA. The possible clinical importance of IVC flow velocity wave forms should be reconsidered in the light of these new anatomical insights.

Acknowledgment. The authors thank J. W. WetselaarWhittaker for preparing the anatomic drawings.

\section{REFERENCES}

1. Reed KL, Appleton CP, Anderson CF, Shenker L, Sahn DJ 1990 Doppler studies of vena cava flows in human fetuses: insight into normal and abnormal cardiac physiology. Circulation 81:498-505

2. Chinn DH, Filly PA, Callen PW 1982 Ultrasonic evaluation of fetal umbilical and hepatic vascular anatomy. Radiology 144:153-157

3. Jeanty P, Romero R, Hobbins JC 1984 Vascular anatomy of the fetus. J Ultrasound Med 3:113-122

4. Balique JG, Regairaz C, Lemeur P, Espalieu Ph, Hugonnier G, Cuilleret J 1984 Anatomical and experimental study of the ductus venosus. Anat Clin $6: 311-316$
5. Moore KL 1977 The cardiovascular system. In: Moore KL (ed) The Developing Human. WB Saunders, Philadelphia, pp 279-283

6. Gilbert SG 1989 The heart, the veins. In: Gilbert SG (ed) Pictorial Human Embryology. University of Washington Press, Seattle, pp 60-78, 98-108

7. Gray H 1985 The veins. In: Clemente CD (ed) Anatomy of the Human Body. Lea \& Febiger, Philadelphia, pp 788-850

8. Huisman TWA, Stewart PA, Wladimiroff JW 1991 Flow velocity waveforms in the fetal inferior vena cava during the second half of normal pregnancy. Ultrasound Med Biol 17:679-682

9. Chan FY, Woo SK, Ghosh A, Tang M, Lam C 1990 Prenatal diagnosis of congenital fetal arrhythmias by simultaneous pulsed Doppler velocimetry of the fetal abdominal aorta and inferior vena cava. Obstet Gynecol 76:200204

10. Campbell S, Wilkin D 1975 Ultrasonic measurement of fetal abdomen circumference in the estimation of fetal weight. Br J Obstet Gynaecol 82:689-697

11. Reuss ML, Rudolph AM, Heymann MA 1981 Selective distribution of microspheres injected into the umbilical veins and inferior venae cavae of fetal sheep. Am J Obstet Gynecol 141:427-432

12. Richter $E 1976$ Rontgenanatomische untersuchungen der nabelvene, des ductus venosus and der pfortader bei menschlichen feten and neugeborenen. Fortschr Rontgenstr 124:552-558

13. Murao F, Senoh D, Takamiya 0, Yamamoto K, Hasegawa K, Kitao M 1989 Ultrasonic evaluation of liver development in the fetus in utero. Gynecol Obstet Invest 28:198-201 